\title{
Interference Analysis for OFDM Transmissions in the Presence of Time-Varying Channel Impairments
}

\author{
Vien Nguyen-Duy-Nhat, Hung Nguyen-Le, Chien Tang-Tan \\ Department of Electronics and Telecommunications Engineering, \\ The University of Danang, University of Science and Technology, Vietnam \\ Correspondence: Hung Nguyen-Le, nlhung@dut.udn.vn \\ Manuscript communication: received 29 August 2012, accepted 4 April 2013
}

\begin{abstract}
This paper is concerned with the detrimental effect of phase noise on the performance of orthogonal frequency division multiplexing (OFDM) transmissions over time-selective channels. In the literature, most of the existing papers analyze the performance of OFDM systems in the presence of either time-selective channels or phase noise. Unlike the existing studies, this paper formulates an approximate expression of signal-to-interference-plus-noise ratio (SINR) at an OFDM receiver in the presence of both phase noise and time-selective channel response. The formulated SINR expression can be used as a guideline in determining appropriate OFDM transmission settings under a given quality-of-service (QoS) requirement. To illustrate the tightness of the approximate SINR formulation, empirical and theoretical values of SINR under different OFDM system settings are presented in this paper.
\end{abstract}

Keywords- Time-selective channel, phase noise, approximate signal-to-interference-plus-noise ratio (SINR) expression, orthogonal frequency division multiplexing (OFDM).

\section{Introduction}

Using the principle of subcarrier orthogonality, orthogonal frequency division multiplexing (OFDM) has offered several superior advantages as compared to the conventional frequency division multiplexing (FDM) technique. As a result, OFDM has been widely recognized as a potential technique for emerging and future mobile broadband communications [1]. Besides its well-known benefits of high spectral-efficiency and robustness against multipath fading propagation, OFDM system performance is, however, vulnerable to timevarying channels (in the presence of moving users) [2] and phase noise (due to phase perturbation in oscillators at transmitter and receiver) [3-8]. In particular, the presence of those channel impairments destroys the orthogonality among subcarriers in OFDM transmissions. The loss of orthogonality would incur significant intercarrier interference (ICI), causing performance degradation in OFDM receivers.

To alleviate the aforementioned detrimental effects, a quantitative relationship between those channel impairments and the resulting ICI power is needed in finding appropriate OFDM transmission settings (that reduce the ICI power). In the literature, several papers [3-10] have derived SINR expressions at an OFDM receiver in the presence of either phase noise or time-varying channels. Specifically, the effect of phase noise has been intensively studied in OFDM transmissions [3-8]. However, the studies have assumed multipath channels to be block-fading (i.e., channel responses are unchanged within one burst duration). Addressing the problem of time-selective channels, $[9,10]$ have formulated closedform expressions of SINR at OFDM receivers by neglecting the effect of phase noise. As a result, the presence of both phase noise and time-varying channel would decrease the accuracy of these existing SINR expressions.

In emerging 4G mobile broadband networks (e.g., LTE-A, WiMAX), there are a large number of highspeed moving nodes (e.g., mobile users in cars and/or trains) communicating with base stations under different quality-of-service (QoS) requirements [11]. For a given QoS level (e.g., SINR values must be greater than a related threshold), the network should determine OFDM transmission parameters to satisfy the QoS level. For instance, by using a theoretical expression of SINR, one can determine the ranges of allowable mobile speeds and/or supported data rates to meet a given QoS level and other system constraints. Hence, an accurate expression of SINR is of importance in determining appropriate OFDM system settings for QoSguaranteed transmissions of broadband data services with high-mobility.

Unlike the aforementioned papers [3-10] considering either high-mobility channel or phase noise in ICI analysis, [12] has included the joint effect of both highmobility channel and phase noise in formulating an exact SINR expression. However, the exact, but complex, SINR formula in [12] is not a closed-form expression. Different from [12], this paper considers the joint effect of both time-varying fading and phase noise in deriving an approximate closed-form expression of SINR by using Taylor series expansion. Under different OFDM 
system settings, this paper provides several numerical results to illustrate the tightness between empirical and analytical values of the approximate SINR expression.

The remaining of this paper is organized as follows. Section 2 describes the formulation of the considered OFDM system in the presence of phase noise and timevarying channels. The detailed steps of approximate SINR formulation are presented in Section 3. Simulation results and relevant discussions are located in Section 4. Finally, Section 5 provides some concluding remarks.

\section{OFDM System Formulation}

\subsection{Transmitted Signal}

This paper considers an uncoded OFDM system where an $N$-point fast Fourier transform (FFT) is employed for the multicarrier transmission as shown in Fig. 1. After inverse FFT (IFFT) and cyclic prefix (CP)

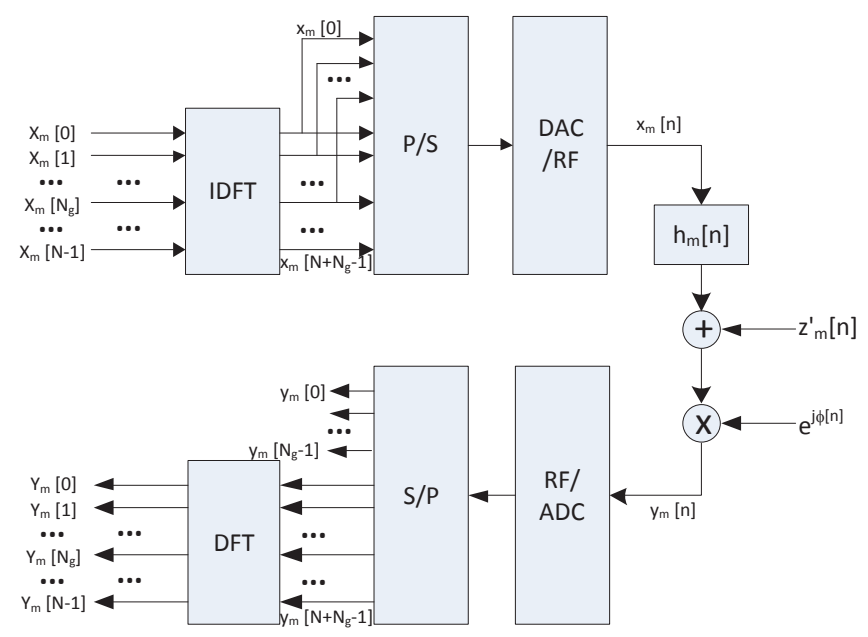

Figure 1. System model

insertion, the transmitted baseband signal of an OFDM symbol can be written as

$$
x_{n}=\frac{1}{\sqrt{N}} \sum_{k=0}^{N-1} X_{k} \exp \left(\frac{j 2 \pi k n}{N}\right),
$$

where $n \in\left\{-N_{g}, \ldots, 0, \ldots, N-1\right\}, N_{g}$ denotes the CP length, $X_{k}$ is the $k$ th data-modulated subcarrier in the considered OFDM symbol.

In the considered channels, the transmitted baseband signal $x_{n}$ in (1) goes through a time-selective channel with phase noise as mathematically described in the next subsection.

\subsection{Doubly Selective Channel and Phase Noise}

For the channel between the transmit antenna and receive antenna, the $l$ th (time-varying) channel tap gain that includes the effect of transmit-receive filters and time-selective propagation is denoted by $h_{l, n}$ where $n$ stands for the index of a time-domain sample. In the considered time-selective channels, possible correlation between channel taps in the spatial dimension is omitted for the sake of simplicity.

As shown in [13], the autocorrelation of the timevarying channel response can be determined by

$$
R_{h}(\tau)=J_{0}\left(2 \pi f_{d} \tau\right),
$$

where $J_{0}($.$) is the zeroth-order Bessel function of the$ first kind, $f_{d}$ is the maximum Doppler frequency, $\tau=$ $n T_{S} / N$, and $T_{S}$ is the useful OFDM symbol length.

Besides time-selective channel propagation, we also consider the effect of phase noise due to the presence of the oscillators' phase perturbation. In the literature, phase noise $\phi(n)$ can be treated as a time-variant multiplicative disturbance [14]

$$
\phi(n)=e^{j \theta(n)} .
$$

In OFDM systems, phase noise appears in freerunning oscillators at both transmitter and receiver. The phase noise process can be modeled as a continuouspath Brownian motion or Wiener process with infinite power [14] which can be formulated as follows:

$$
\theta(n)=\theta(n-1)+\varepsilon(n)
$$

where $\theta(0)=0$ and $\varepsilon(n)$ denotes Gaussian distributed random variables with zero-mean and variance of $\sigma^{2}=$ $2 \pi \beta T_{s}, \beta$ stands for the phase noise linewidth (i.e, frequency spacing between $3 \mathrm{~dB}$ points in its Lorentzian power spectral density of the local oscillator). The phase noise processes under different values of $\beta T_{S}$ are depicted in Fig. 2. As we can see, the larger value of $\beta T_{S}$, the more adverse effect of phase noise.

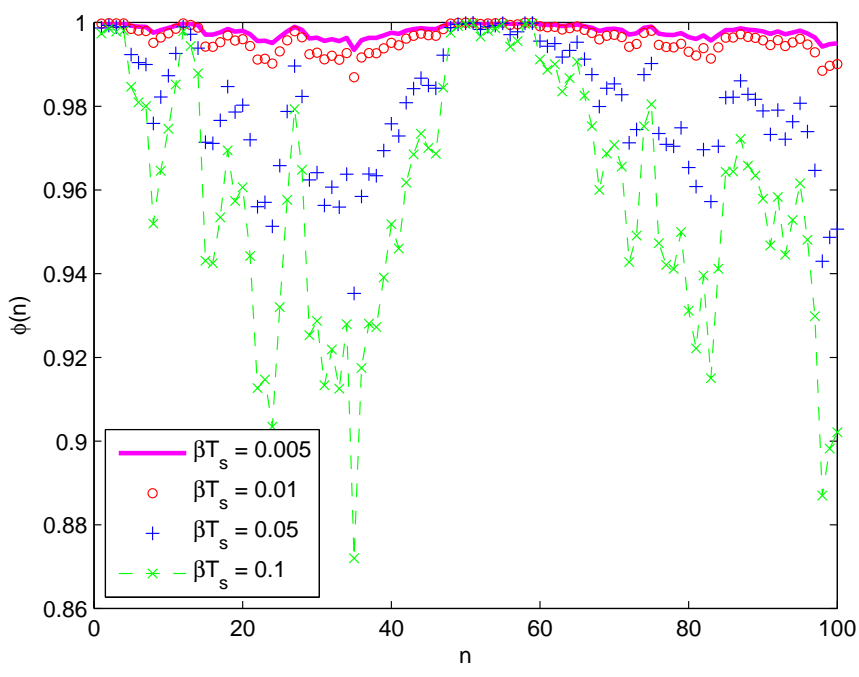

Figure 2. Illustration of phase noise process

As shown in [15], the autocorrelation function of $\phi(t)$ can be computed by

$$
R_{\phi}(\tau)=e^{-\pi \beta|\tau|} .
$$

\subsection{Received Signal Model}

In the presence of both time-selective channel and phase noise, the $n$th received sample in an OFDM 
symbol (after CP removal) can be represented by

$$
y_{n}=e^{j \theta(n)} \sum_{l=0}^{L-1} h_{l, n} x_{n-l}+z_{n}
$$

where $n=0, \ldots, N-1$ and $z_{n}$ is the additive white Gaussian noise (AWGN) with variance $N_{o}$. In this paper, the powers of both the transmitted signals and channel impulse response (CIR) are normalized to one and the resulting signal-to-noise ratio (SNR) can be determined by $S N R=\frac{1}{N_{0}}$.

As observed in (6), the presence of phase noise introduces a time-domain phase rotation that will translate into ICI in the frequency domain as presented by the next formulations. In addition, the time-variation of the multipath channels also induces ICI in the frequency domain [16]. Consequently, the presence of both phase noise and time-selective channels would incur significant ICI power at an OFDM receiver, giving rise to a an irreducible error floor in the receiver performance.

After performing FFT at the receiver, the frequencydomain signals can be determined by

$$
Y_{k}=C_{0} H_{k} X_{k}+I_{k}+Z_{k}
$$

where $I_{k}=\sum_{p=1}^{N-1} C_{p} H_{k-p} X_{k-p}$ denotes inter-carrierinterference (ICI), $C_{p}=\frac{1}{N} \sum_{n=0}^{N} e^{-\frac{j 2 \pi n p}{N}} e^{j \theta(n)}$, and $H_{k}=$ $\frac{1}{N} \sum_{n=0}^{N-1} \sum_{l=0}^{L-1} h_{l, n} e^{-j 2 \pi k l / N}$.

Based on (7), the formulation of an approximate SINR expression is derived in the next section.

\section{InTERfEREnCE ANALYsis}

\subsection{SINR Formulation}

In (7), the power of the $k$ th subcarrier at the receiver can be expressed by

$$
P_{r}=P_{\text {des }}+P_{I C I}+N_{0},
$$

where $P_{r}=E\left[\left|Y_{k}\right|^{2}\right], P_{d e s}=E\left[\left|C_{0} H_{k} X_{k}\right|^{2}\right], P_{I C I}=$ $E\left[\left|I_{k}\right|^{2}\right]$, and $N_{0}=E\left[\left|Z_{k}\right|^{2}\right]$.

Hence, the value of signal-to-interference-plus-noise ratio SINR can be determined by

$$
\mathrm{SINR}=\frac{P_{\text {des }}}{P_{I C I}+N_{0}} .
$$

In (9), the ICI power can be computed by

$$
P_{I C I}=\int_{-1}^{1}(1-|x|)\left(1-R\left(T_{s} x\right)\right) d x,
$$

where $R\left(T_{S} x\right)$ denotes the autocorrelation function of the effective channel response [10].

It is assumed that the power of phase noise and fading have been normalized to one, i.e. $E\left[\sum_{p=0}^{N-1}\left|C_{p}\right|^{2}\right]=1$, and $E\left[\sum_{p=0}^{N-1}\left|H_{k-p}\right|^{2}\right]=1$, respectively. In (9), the power of the desired signal can be calculated by

$$
P_{\text {des }}=E\left[\left|C_{0}\right|^{2}\left|H_{k}\right|^{2}\right]=\left(1-P_{\text {ICIpn }}\right)\left(1-P_{\text {ICIch }}\right)
$$

where

$$
P_{\text {ICI } p}=E\left[\sum_{p=1}^{N-1}\left|C_{p}\right|^{2}\right],
$$

and

$$
P_{\text {ICIch }}=E\left[\sum_{p=1}^{N-1}\left|H_{k-p}\right|^{2}\right] .
$$

By using (2), (5), and (10), one can obtain

$$
\begin{aligned}
P_{\text {ICI } n} & =\int_{-1}^{1}(1-|x|)\left(1-R_{\phi}\left(T_{s} x\right)\right) d x \\
& =\frac{2-2 e^{-\pi T_{s} \beta}-2 \pi \beta T_{s}+\pi^{2} \beta^{2} T_{s}^{2}}{\pi^{2} \beta^{2} T_{s}^{2}},
\end{aligned}
$$

and

$$
\begin{aligned}
P_{\text {ICICh }} & =\int_{-1}^{1}(1-|x|)\left(1-R_{h}\left(T_{s} x\right)\right) d x \\
& \approx \frac{0 F_{1}\left[2,-\left(\pi f_{d} T_{s}\right)^{2}\right]}{\Gamma(2)} \\
& -2_{p} F_{q}\left[\left\{\frac{1}{2}\right\},\left\{1, \frac{3}{2}\right\},-\left(f_{d} \pi T_{s}\right)^{2}\right],
\end{aligned}
$$

where ${ }_{0} \boldsymbol{F}_{\mathbf{1}}(a, z), \boldsymbol{\Gamma}(a)$, and ${ }_{p} \boldsymbol{F}_{q}(a ; b ; z)$ are the regularized confluent hypergeometric function, gamma function, and generalized hypergeometric function, respectively.

From (10), (12), and (13), we obtain

$$
\begin{aligned}
P_{\text {des }} & =\left(1+\frac{2-2 e^{-\pi T_{s} \beta}-2 \pi \beta T_{s}+\pi^{2} \beta^{2} T_{s}^{2}}{\pi^{2} \beta^{2} T_{s}^{2}}\right) \\
& \times\left(1-\frac{{ }_{0} F_{1}\left[2,-\left(\pi f_{d} T_{s}\right)^{2}\right]}{\Gamma(2)}\right. \\
& \left.-2_{p} F_{q}\left[\left\{\frac{1}{2}\right\},\left\{1, \frac{3}{2}\right\},-\left(\pi f_{d} T_{s}\right)^{2}\right]\right) .
\end{aligned}
$$

By assuming fading channel response and phase noise are statistically independent, one can deduce

$$
R\left(T_{s} x\right)=R_{\phi}\left(T_{s} x\right) R_{h}\left(T_{s} x\right) .
$$

Based on (2), (5), (10) and (15), we can obtain

$$
\begin{aligned}
P_{I C I} & =\frac{1}{3}\left(3+3 \frac{{ }_{0} \boldsymbol{F}_{\mathbf{1}}\left[2,-\left(\pi f_{d} T_{s}\right)^{2}\right]}{\Gamma(2)}\right. \\
& +3 \pi \beta T_{s} \frac{{ }_{0} \boldsymbol{F}_{\mathbf{1}}\left[2,-f_{d}^{2} \pi^{2} T_{s}^{2}\right]}{\Gamma(2)} \\
& -6_{p} \boldsymbol{F}_{q}\left[\frac{1}{2}, 1, \frac{3}{2},-\left(\pi f_{d} T_{s}\right)^{2}\right] \\
& \left.-2 \pi \beta T_{s p} F_{q}\left[\frac{3}{2}, 1, \frac{5}{2},-\left(\pi f_{d} T_{s}\right)^{2}\right]\right) .
\end{aligned}
$$

Substituting (14), (16) into (9), we can obtain a closeform expression of SINR that considers the joint effect of time-varying channel and phase noise. However, the closed-form SINR expression is relatively complicated. In this paper, we explore Taylor series expansion to obtain an approximate expression of SINR as presented in the next subsection. 


\subsection{Approximate SINR Formulation}

In order to separate the signal and noise terms, applying the third-order Taylor series expansion of $e^{-\pi \beta T_{s}}$, we obtain

$$
e^{-\pi \beta T_{s}} \approx 1-\pi \beta T_{S}+\frac{\left(\pi \beta T_{S}\right)^{2}}{2}-\frac{\left(\pi \beta T_{S}\right)^{3}}{6}
$$

From (12), we have

$$
P_{I C I p n} \approx \frac{\pi \beta T_{s}}{3} .
$$

Applying Taylor series expansion for (13) to third-order terms, we get

$$
P_{\text {ICIch }} \approx \frac{\left(\pi f_{d} T_{s}\right)^{2}}{6}-\frac{\left(\pi f_{d} T_{s}\right)^{4}}{60}+\frac{\left(\pi f_{d} T_{s}\right)^{6}}{1008} .
$$

Similarly, one can rewrite (14) by

$$
\begin{aligned}
P_{\text {des }} & \approx\left(1-\frac{\pi \beta T_{s}}{3}\right)\left(1-\frac{\left(\pi f_{d} T_{s}\right)^{2}}{6}-\right. \\
& \left.-\frac{\left(\pi f_{d} T_{s}\right)^{4}}{60}+\frac{\left(\pi f_{d} T_{s}\right)^{6}}{1008}\right) .
\end{aligned}
$$

Hence, the approximate ICI power can be calculated as

$$
\begin{aligned}
\text { PICIapprox }_{2} & \frac{\pi \beta T_{s}}{3}+\frac{\left(\pi f_{d} T_{s}\right)^{2}}{3}\left(\frac{1}{2}-\frac{3 \pi \beta T_{s}}{10}\right)+ \\
& +\frac{\left(\pi f_{d} T_{s}\right)^{4}}{3}\left(-\frac{1}{20}+\frac{\pi \beta T_{s}}{28}\right)+ \\
& +\frac{\left(\pi f_{d} T_{s}\right)^{6}}{3}\left(\frac{1}{336}-\frac{\pi \beta T_{s}}{432}\right) .
\end{aligned}
$$

Using (9), (21), (19), and (21) and first-order terms in Taylor series, we can obtain a SINR expression as a function of the normalized Doppler shift $f_{d} T_{s}$, and the product of the $3 \mathrm{~dB}$ two-side phase noise linewidth $\beta$ and the data symbol period $T_{s}$. In particular, the approximate expression of SINR can be expressed by

$$
\operatorname{SINR}_{\text {approx }} \approx \frac{\left(1-\frac{\psi}{3}\right)\left(1-\frac{\vartheta^{2}}{6}\right)}{\frac{\psi}{3}+\frac{\vartheta^{2}}{3}\left(\frac{1}{2}-\frac{3 \psi}{10}\right)+N_{0}},
$$

where $\psi=\pi \beta T_{s}$, and $\vartheta=\pi f_{d} T_{s}$.

In particular, for BPSK, 16-QAM, and 64-QAM modulations with coherent detection and Gray encoding, the BER performance over Rayleigh fading channels can be given by [17]

$$
\begin{aligned}
& \mathrm{BER}_{B P S K}^{\text {Ray }}=\frac{1}{2}\left(1-\frac{1}{\sqrt{1+\frac{1}{\operatorname{SINR}}}}\right), \\
& \mathrm{BER}_{16-Q A M}^{\text {Ray }}=\frac{3}{8}\left(1-\frac{1}{\sqrt{1+\frac{5}{2 \operatorname{SINR}}}}\right) \text {, } \\
& \mathrm{BER}_{64-Q A M}^{\text {Ray }}=\frac{7}{24}\left(1-\frac{1}{\sqrt{1+\frac{7}{\operatorname{SINR}}}}\right),
\end{aligned}
$$

where $\mathrm{BER}_{B P S K}^{\text {Ray }}$, $\mathrm{BER}_{16-\mathrm{Q} A M^{\prime}}^{\text {Ray }}$ and $\mathrm{BER}_{64-Q A M}^{\text {Ray }}$ are the BER performance for BPSK, 16-QAM, and 64-QAM modulations, respectively.

Based on the above approximate SINR expression (22), one can deduce the approximate expressions of bit error rate (BER) values of the considered OFDM system by using related BER formulations in (23)-(25).

\section{Simulation Results and Discussions}

Following the WiMAX system settings [18], computer simulation was conducted to evaluate the tightness of the derived approximate SINR expression for OFDM systems over time-selective channels with phase noise. The time-varying multipath fading channel with the number of channel tap gains $L=10$ and the exponentially decaying power-delay profile [19] is generated by using Jakes' model [13]. Unless otherwise stated, the considered mobile terminal has a speed $v=100 \mathrm{~km} / \mathrm{h}$ and operating at $f_{c}=3.5 \mathrm{GHz}$. Each OFDM symbol uses a 512-point FFT with sampling frequency $f_{s}=5.6 \mathrm{MHz}$ and a CP length of 40 samples $\left(N_{g}=40\right)$ [18]. For each transmission burst (of each simulation trial), the phase noise is a random process following a continuous-path Brownian motion [14].

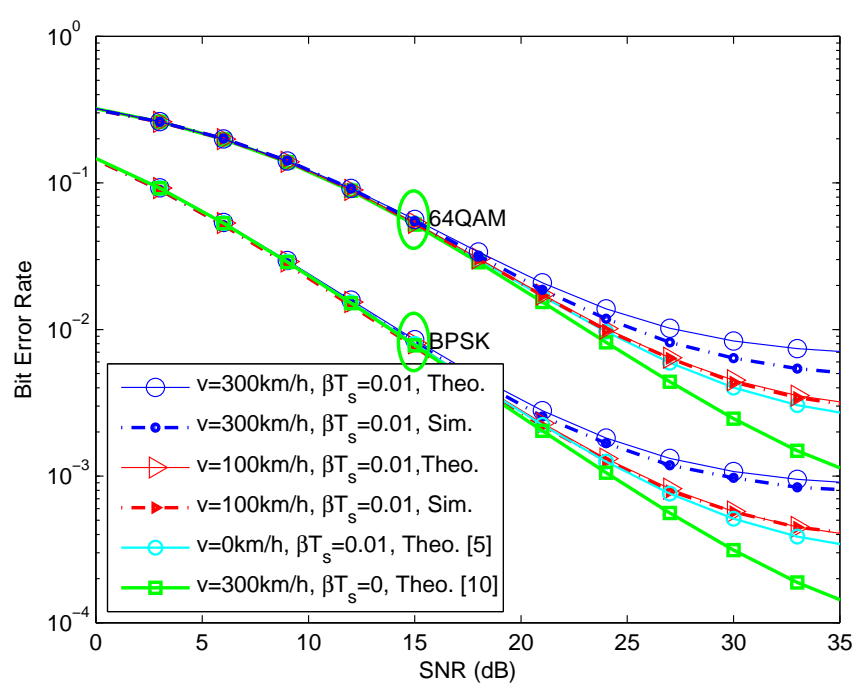

Figure 3. BER analysis for BPSK and 64-QAM signal versus SNR for the mobile terminal speed of $100 \mathrm{~km} / \mathrm{h}$ and $300 \mathrm{~km} / \mathrm{h}$ with $\beta T_{S}=0.01$

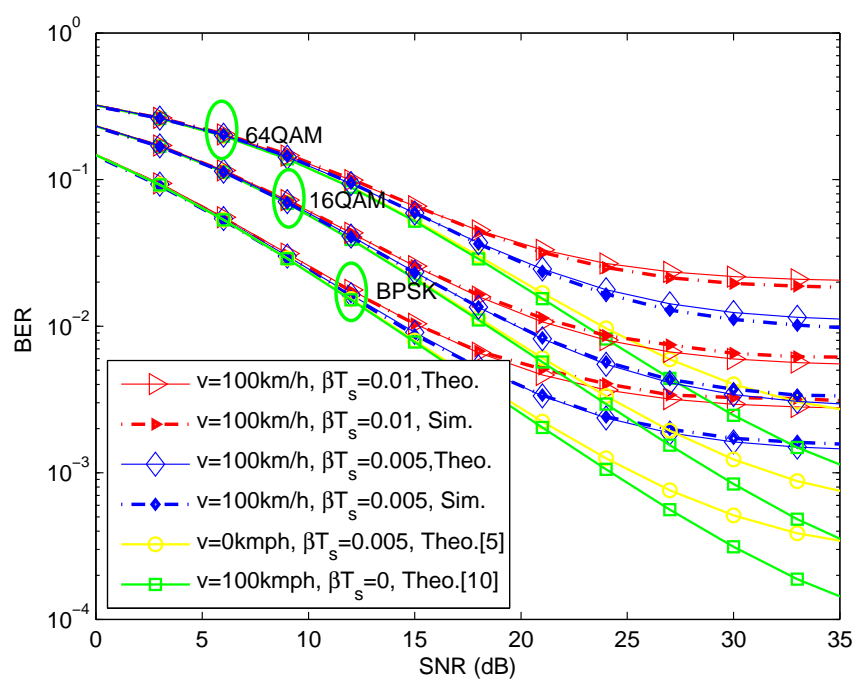

Figure 4. BER analysis for BPSK, 16-QAM, and 64-QAM signal versus SNR for the mobile terminal speed of $100 \mathrm{~km} / \mathrm{h}$ with $\beta T_{S}=0.01$, and 0.005

To investigate BER performance of OFDM transmissions under different scenarios, Figs. 3 and 4 shows the simulated and theoretical BER values versus mo- 


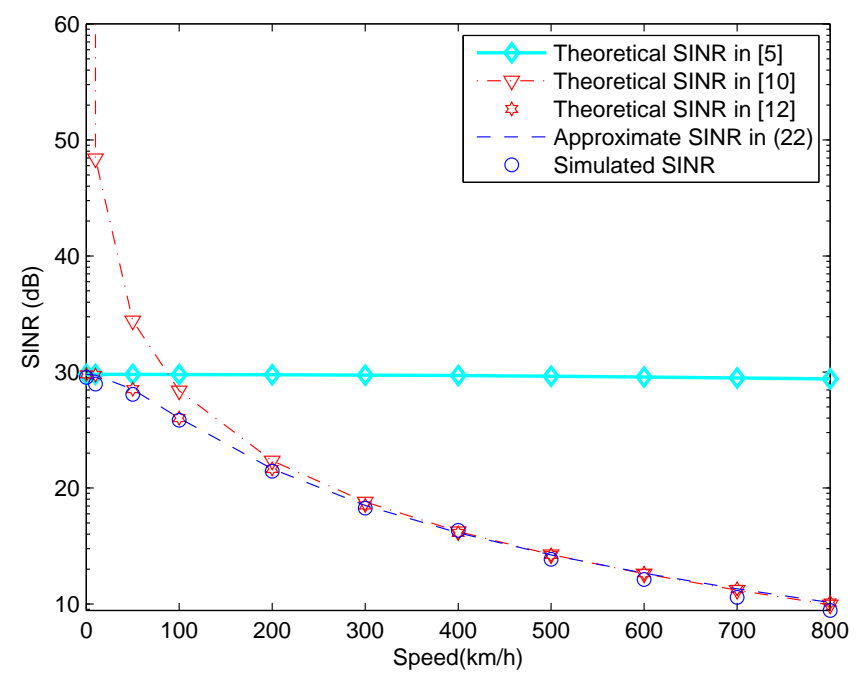

Figure 5. The numerical results of SINR versus mobile speeds

bile speed and SNR, under different modulations. In particular, the theoretical BER values were obtained by using approximate BER expressions in Section 3.2. As observed, the theoretical BER values are close to the simulated ones (as shown by dash and solid curves). In addition, the numerical results illustrate that the presence of phase noise and time-varying channels induces significant BER performance degradation as mobile speed and phase noise level $\beta T_{S}$ increase.

To verify the accuracy of the derived approximate SINR expression, Fig. 5 shows the simulated and theoretical SINR values versus mobile user speed under the phase noise level of $\beta T_{s}=0.01$. In [5], the theoretical SINR expressions have been derived by ignoring the effect of time-varying channels. Therefore, the theoretical SINR values of [5] are very close to the simulated SINR values under the condition of low mobile speeds (e.g., $<100 \mathrm{~km} / \mathrm{h}$ ). As the mobile speed increases (e.g., $>100 \mathrm{~km} / \mathrm{h}$ ), the gap between the simulated and theoretical SINR values (of [5]) increase. Taking into account the effect of time-varying channels, [9] has derived the theoretical SINR expression by neglecting the effect of phase noise. As a result, the theoretical SINR values of [9] are very close to the simulated ones under the condition of high-mobility (e.g., $>300$ $\mathrm{km} / \mathrm{h}$ ). However, the gap between the simulated and theoretical SINR values (of [9]) increase as the mobile speed reduces (e.g., $<300 \mathrm{~km} / \mathrm{h}$ ) due to the dominant ICI power induced by phase noise (that has been ignored in [9]). As observed, the derived approximate SINR value in (22) and exact SINR in [12] are very close together under any mobile user speed. For low computational complexity, one can use the approximate SINR (22) with a closed-form expression.

Unlike [5] and [9], this paper formulates an approximate SINR expression by taking into account the joint effect of both phase noise and time-varying channels. As a results, the simulated SINR values and (approximate) theoretical ones of (22) are in good agreement for any value of mobile speed and phase noise as showed in Fig. 6.

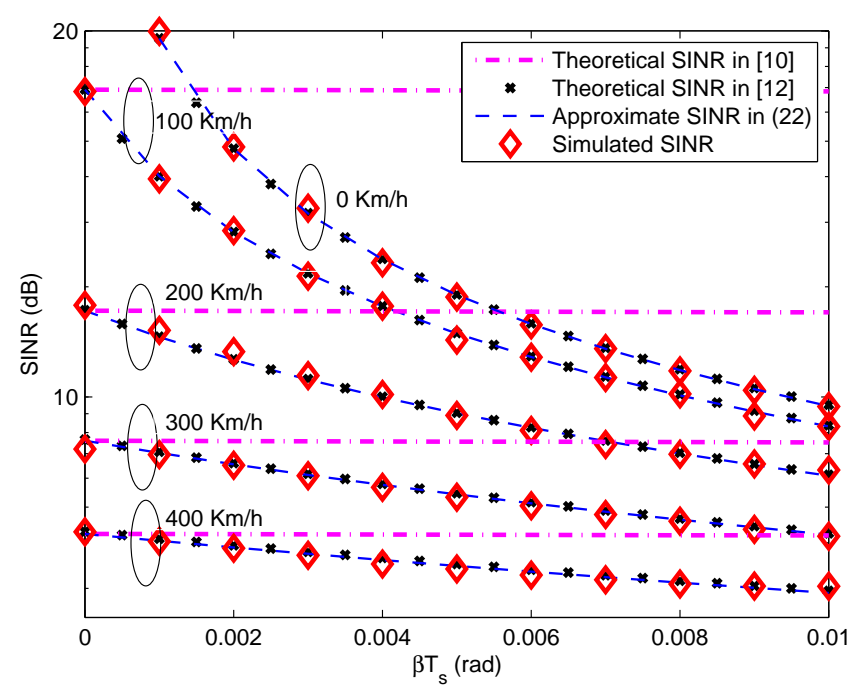

Figure 6. The analytical and empirical results of SINR versus phase noise for the differential mobile speeds.

In addition, the numerical results of the derived approximate SINR expression (22) can be used as guidelines in determining the maximum allowable mobile speed under a given $\mathrm{QoS}$ requirement (i.e., SINR values must be greater than a related threshold). Figs. 7 the SINR versus SNR is plotted for different mobile speeds. To achieve the SINR $>20 \mathrm{~dB}$, the SNR should be more than $22 \mathrm{~dB}$ with a mobile speed of $100 \mathrm{~km} / \mathrm{h}$ and the SINR requirement can not be satisfied if SNR is less than $30 \mathrm{~dB}$ for mobile speeds of $300 \mathrm{~km} / \mathrm{h}$ and 500 $\mathrm{km} / \mathrm{h}$.

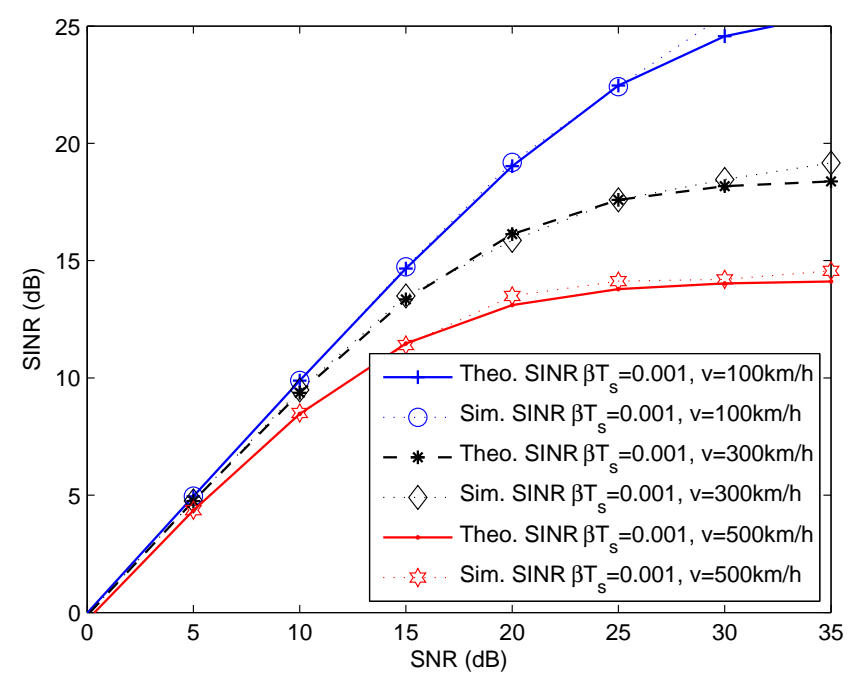

Figure 7. SINR versus SNR when $\beta T_{s}=0.001, v=100,300,500 \mathrm{~km} / \mathrm{h}$

The SINR curves at mobile speeds of $100 \mathrm{~km} / \mathrm{h}$ and $350 \mathrm{~km} / \mathrm{h}$ under radio carrier frequencies of $3.5 \mathrm{GHz}$, $5 \mathrm{GHz}$, and $10 \mathrm{GHz}$ are show in Fig. 9. As can be seen from this figure, the degradation increases as the carrier frequency increase. It can also be seen that the carrier frequency of $10 \mathrm{GHz}$ is inappropriate with high speed applications.

For a given QoS requirement, the approximate SINR expression also helps to determine allowable data rates of provided services. In particular, Fig. 8 shows nu- 


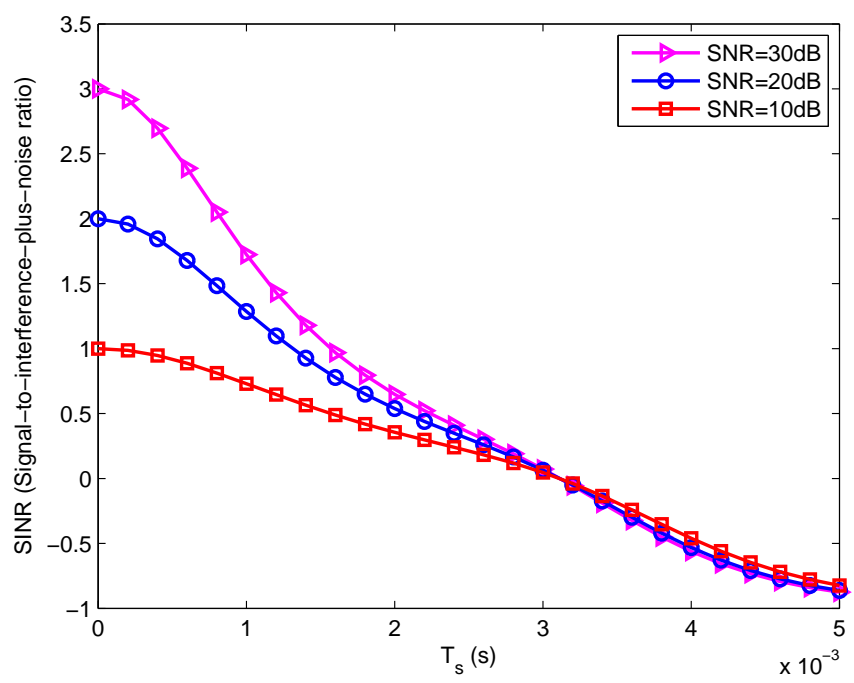

Figure 8. SINR versus $T_{s}$ when $\beta T_{s}=0.01$, speed of $100 \mathrm{~km} / \mathrm{h}$

merical SINR results of (22) versus the OFDM symbol length $T_{s}$. Specifically, by using the approximate SINR expression (22), one can determine that the values of $T_{S}$ should be smaller than a certain threshold so that the actual SINR values are greater than a required value (corresponding to the required QoS level).

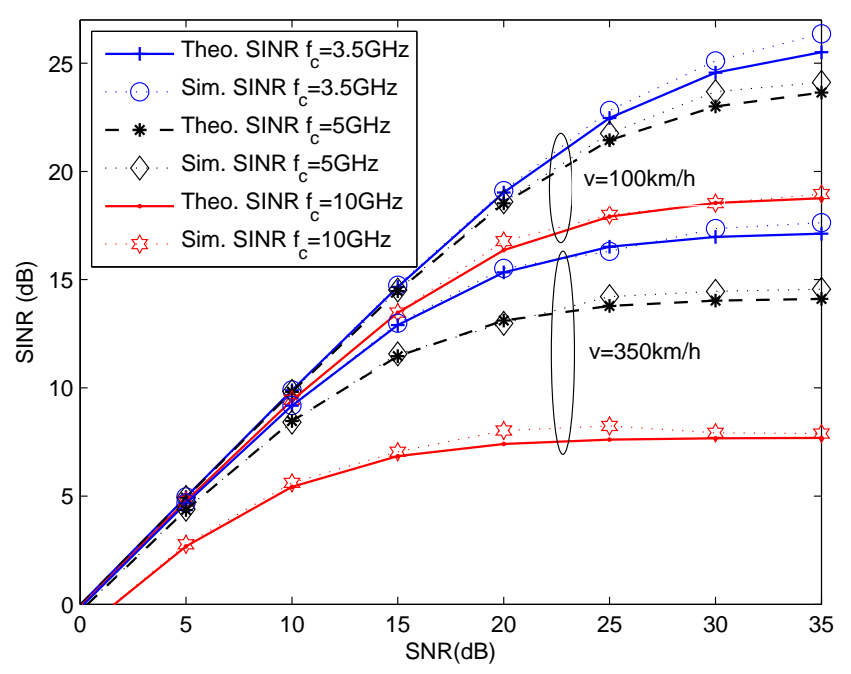

Figure 9. The approximate SINR (22) and related simulated values versus SNR when $\beta T_{s}=0.001, v=100 \mathrm{~km} / \mathrm{h}, f_{c}=3.5 \mathrm{GHz}, 5 \mathrm{GHz}$, and $10 \mathrm{GHz}$

Table I

LTE AND WIMAX BAND 5

\begin{tabular}{|l|c|c|}
\hline & LTE [20] & WiMAX [18] \\
\hline System channel bandwidth $(\mathrm{MHz})$ & 5 & 5 \\
\hline Sampling frequency $F_{S}(\mathrm{MHz})$ & 7.68 & 5.6 \\
\hline FFT size $N_{f f t}$ & 512 & 512 \\
\hline Subcarrier frequency spacing $(\mathrm{kHz})$ & 15 & 10.94 \\
\hline Useful symbol duration $T_{s}(\mu s)$ & 66.7 & 91.4 \\
\hline
\end{tabular}

In Fig. 10, SINRs are plotted versus SNR under LTE and WiMAX system settings as shown in Table 1. The SINR values of the considered LTE system are higher than those of the considered WiMAX system under the

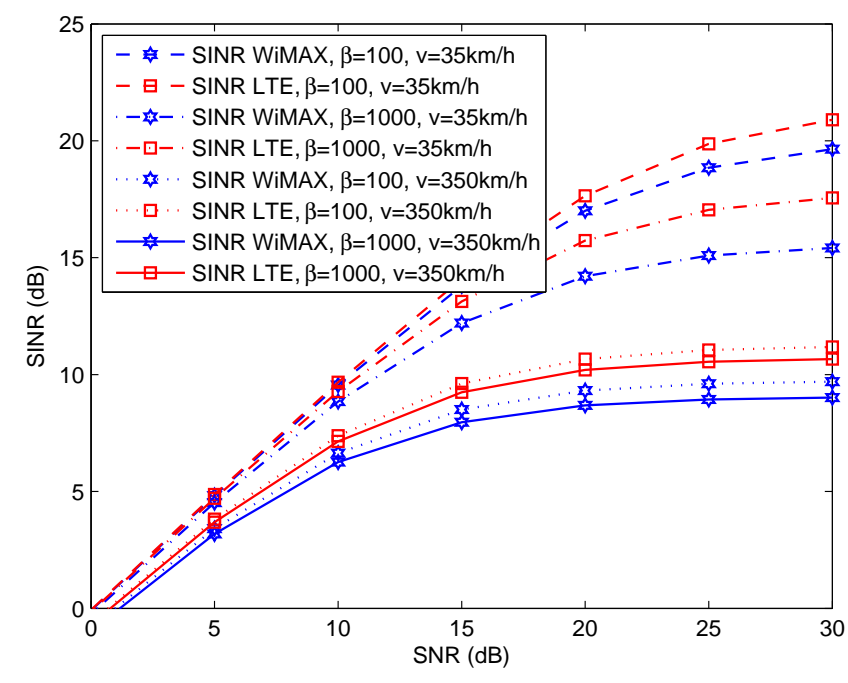

Figure 10. SINR comparison between the LTE and WiMAX system settings

same $\beta$ and mobile speed values since $T_{S}$ of the LTE setting is greater than that of the WiMAX one.

\section{Conclusion}

This paper developed an approximate SINR expression for OFDM transmissions in the presence of both phase noise and time-varying channels. Unlike other existing SINR formulations, the closed-form expression of approximate SINR in this paper offers a better agreement with empirical SINR values over a wide range of mobile speeds. The derived SINR formulation can be used as theoretical guidelines in choosing appropriate OFDM transmission settings under a given QoS requirement and related system constraints.

\section{REFERENCES}

[1] G. L. Stuber, J. R. Barry, S. W. Mclaughlin, Y. Li, M. A. Ingram, and T. G. Pratt, "Broadband mimo-ofdm wireless communications," Proceedings of the IEEE, vol. 92, no. 2, pp. 271-294, 2004.

[2] Z. Tang, R. C. Cannizzaro, G. Leus, and P. Banelli, "Pilot-assisted time-varying channel estimation for ofdm systems," IEEE Transactions on Signal Processing, vol. 55, no. 5, pp. 2226-2238, 2007.

[3] L. Piazzo and P. Mandarini, "Analysis of phase noise effects in ofdm modems," IEEE Transactions on Communications, vol. 50, no. 10, pp. 1696-1705, 2002.

[4] T. Pollet, M. Van Bladel, and M. Moeneclaey, "Ber sensitivity of ofdm systems to carrier frequency offset and wiener phase noise," IEEE Transactions on Communications, vol. 43, no. 234, pp. 191-193, 1995.

[5] P. Robertson and S. Kaiser, "Analysis of the effects of phase-noise in orthogonal frequency division multiplex (ofdm) systems," in 1995 IEEE International Conference on Communications, ICC'95 Seattle,'Gateway to Globalization', vol. 3. IEEE, 1995, pp. 1652-1657.

[6] L. Tomba, "On the effect of wiener phase noise in ofdm systems," IEEE Transactions on Communications, vol. 46, no. 5, pp. 580-583, 1998.

[7] S. Wu and Y. Bar-Ness, "Ofdm systems in the presence of phase noise: consequences and solutions," IEEE Trans- 
actions on Communications, vol. 52, no. 11, pp. 1988-1996, 2004.

[8] L. Rugini and P. Banelli, "Ber of ofdm systems impaired by carrier frequency offset in multipath fading channels," IEEE Transactions on Wireless Communications, vol. 4, no. 5, pp. 2279-2288, 2005.

[9] P. Robertson and S. Kaiser, "The effects of doppler spreads in ofdm (a) mobile radio systems," in Vehicular Technology Conference, 1999. VTC 1999-Fall. IEEE VTS 50th, vol. 1. IEEE, 1999, pp. 329-333.

[10] Y. Li and L. J. Cimini Jr, "Bounds on the interchannel interference of ofdm in time-varying impairments," IEEE Transactions on Communications, vol. 49, no. 3, pp. 401404, 2001.

[11] M. Alasti, B. Neekzad, J. Hui, and R. Vannithamby, "Quality of service in wimax and lte networks [topics in wireless communications]," IEEE Communications Magazine, vol. 48, no. 5, pp. 104-111, 2010.

[12] Y. Zhang and H. Liu, "Mimo-ofdm systems in the presence of phase noise and doubly selective fading," IEEE Transactions on Vehicular Technology, vol. 56, no. 4, pp. 2277-2285, 2007.

[13] Y. R. Zheng and C. Xiao, "Simulation models with correct statistical properties for rayleigh fading channels," IEEE Transactions on Communications, vol. 51, no. 6, pp. 920-928, 2003.

[14] S. Wu and Y. Bar-Ness, "A phase noise suppression algorithm for ofdm-based wlans," IEEE Communications Letters, vol. 6, no. 12, pp. 535-537, 2002.

[15] S. Wu, P. Liu, and Y. Bar-Ness, "Phase noise estimation and mitigation for ofdm systems," IEEE Transactions on Wireless Communications, vol. 5, no. 12, pp. 3616-3625, 2006.

[16] C. Shin, J. G. Andrews, and E. J. Powers, "An efficient design of doubly selective channel estimation for ofdm systems," IEEE Transactions on Wireless Communications, vol. 6, no. 10, pp. 3790-3802, 2007.

[17] S. Sampei, Applications of digital wireless technologies to global wireless communications. Prentice Hall PTR, 1997.

[18] W. F. Proprietary, "Wimax forum $(\mathbb{B}$ air interface specifications," WiMAX Forum, WMF-T23-001-R020v01, 2011.

[19] T. Zemen and C. F. Mecklenbrauker, "Time-variant channel estimation using discrete prolate spheroidal sequences," IEEE Transactions on Signal Processing, vol. 53, no. 9, pp. 3597-3607, 2005.

[20] 3GPP, "Overview of 3gpp release 6: Summary of all release 6 features (version tsg 33)," 3GPP Rel-6, 2006.

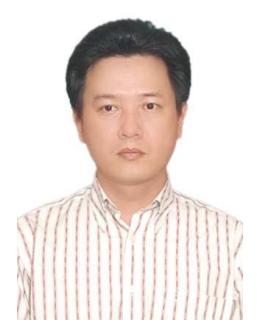

Vien Nguyen-Duy-Nhat received the B.Eng. degree in Electronic Engineering from Danang University of Technology, (DUT), Vietnam, in July 1997. In September 1997, he joined the Department of Electronics and Telecommunications Engineering, Danang University of Technology. In 2003, he received the M.Eng. degree in Electrical Engineering from Ho Chi Minh City University of Technology (HCMUT), Vietnam. His research interests include signal processing algorithms for wireless systems, multiple-input multiple-output (MIMO) systems, multi-users and multihop wireless communications.

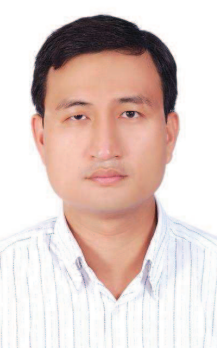

Hung Nguyen-Le received the B.Eng. degree and the M.Eng. degree in Electrical Engineering from Hochiminh City University of Technology, Vietnam, in 2001 and 2003, respectively. He obtained the Ph.D. degree in Electrical Engineering from the National University of Singapore in 2008. From 2008 to 2009 he worked as a Postdoctoral Research Fellow in the Department of Electrical and Computer Engineering, McGill University, Montreal, Canada. Since 2010, he has been with the Department of Electronics and Telecommunications Engineering the University of Danang, University of Science and Technology. His research interests include array signal processing, multiuser/multicell transmissions, channel estimation and synchronization in broadband wireless communications.

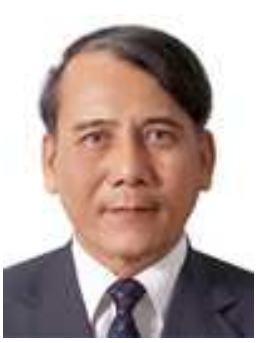

Chien Tang-Tan received the Bachelor degree in Physics - Electronics from the Unversity of Science, HCMC, Vietnam, in 1979; he joined the Department of Electronics and Telecommunications Engineering, Danang University of Technology. In 2003, he received the PhD. Eng. degree in Telecom. Engineering from Hanoi University of Technology, Vietnam. He was promoted to Associate Professor in 2010. His research interests include Electromagnetic Compatibility (EMC) and processing signals for Wireless Communications. 